one-tenth candle at one kilometre is just sufficient to excite vision. When a sector was rotated in front of the pinhole, a source 16 times as strong as this acting for $\frac{1}{1 \frac{1}{2}}$ sec. or $\frac{1}{60}$ sec. according to the observer was sufficient to excite vision.

One-tenth candle at one kilometre delivers about $4.51 \times 10^{-5}$ erg per sec. on a pupil of $45 \mathrm{sq} . \mathrm{mm}$. area. Only about 1 per cent of this lies between $410 \mu \mu$ and $760 \mu \mu$. If we assume that the source has the same energy distribution as that of a black body at $2000^{\circ} \mathrm{K}$, and use Hecht's values for the visibility of light to the dark adapted eye, we can obtain the distribution of the light of the source over the spectrum. About 0.21 of the whole light lies in the range $520-540 \mu \mu$, and about 0.017 of the energy between 410 and $760 \mu \mu$ lies within this range. The quantum at $530 \mu \mu$ has the value $3 \cdot 71 \times 10^{-12} \mathrm{erg}$. see. This gives the result that 9840 quanta per sec. of green light are required to produce the sensation of a continuous point source. For a flash to be visible 1400 or 2600 quanta of green light are necessary, according to the observer. If the flash is red or violet, a much greater number of quanta will be required. In making the calculation we have neglected the absorption of light in the media of the eye, but this cannot affect the result seriously.

There is a striking result connected with the visibility of small sources : visibility requires the same quantity of light, no matter what is the angle subtended by the source, up to an angle of one or two degrees. This is known as Riccò's law of foveal vision. It might be supposed that there is some connexion between this law and the quantum. But the numerical values show that there is none.

R. A. Houstoun. JAS. F. SHEARER.

University of Glasgow, July 24.

\section{Effect of Magnetic Field on Dielectrics.}

IN a letter in the issue of NATuRE for July 12, Prof P. L. Burns states that the power factor of certain dielectrics is decreased when a constant magnetic field is superimposed on the dielectric which is being subjected to an alternating electric stress. One would expect that on the Debye dipole theory of polar molecules in a viscous medium the power factor would be changed by a magnetic field.

The motion of the electrical charges, or, if the molecule contained a magnetic moment, the motion of the magnetic field in a superimposed electrical or magnetic field, would give rise to a loss of energy. The force acting on the molecule would be of a nature similar to the frictional force due to the viscosity of the medium. The relaxation time is given approximately by $4 \pi \eta r^{3} / K T$. These forces could be considered to increase the value of $\eta$ and hence increase the relaxation time. This would tend to shift the peak of the power factor against frequency curve toward lower frequencies. Therefore, depending on which side of the peak one was measuring the power factor, the power factor would be either increased or decreased by the magnetic field.

It would be interesting to know on what materials Prof. Burns found his results. It seems clear that power loss in dielectrics cannot be completely explained on the Debye theory. Some work has been done here considering the double layer surrounding colloidal particles and its effect on the power factor. Possibly these experiments of Prof. Burns would make it possible to distinguish between the two mechanisms.

Experimental Station JoHN B. MILES, JR.

E. I. du Pont de Nemours and Company, Wilmington, Delaware, Aug. 12.
The Space-Group of Strychnine.

IN connexion with the recent work on strychnine described by Prof. $R$. Robinson in his recent Bakerian lecture, the results obtained from an $\mathrm{X}$-ray investigation may prove of interest.

The substance forms extremely good crystals, for which I am very much indebted to Prof, Robinson. Results :

Unit cell $\quad a=11 \cdot 9_{2} \mathrm{~A} . \quad b=12 \cdot 1_{3} \mathrm{~A} . \quad c=11 \cdot 3_{0} \mathrm{~A}$.

Axial ratio $\quad a \quad: b \quad: \quad c$

\begin{tabular}{ccccc}
\multicolumn{1}{c}{$a$} & $:$ & $b$ & $:$ & \multicolumn{1}{c}{$c$} \\
0.983 & $:$ & 1 & $:$ & 0.931 \\
0.9827 & $:$ & 1 & $:$ & 0.9309
\end{tabular}

No. of molecules 4

Space group $\quad Q_{4} \quad$ (Astbury and Yardley, Phil. Trans., A, vol. 224; 1924).

The dimensions of the cell, and the space group, are such that four molecules of the disc-shaped form suggested by Prof. Robinson can be fitted into the unit cell. Owing to the complicated nature of the molecule, and to the fact that it is composed of comparatively light atoms which have approximately equal scattering power for $\mathrm{X}$-rays, it is impossible at this stage to make any further assumptions as to its structure.

The Davy Faraday Laboratory,

Thora C. Marwick.

\title{
Occurrence of Mannitol in Spike Disease of Santalum album (Linn.).
}

WHILE investigating the water soluble constituents of the spiked leaf of sandal, it was found that crystals of mannitol separated on slowly evaporating the extract after clarification with basic lead acetate. 2-3 per cent of the alcohol, calculated on the weight of the green material, has been found in all the samples so far examined (15), while in no case has it been detected in healthy samples. The significance of this fact at the present stage is difficult to understand, but it appears that mannitol is one of the metabolic products of the virus. Whether such characteristic products are formed during the course of other wellknown virus diseases or not is a matter for future investigation, and workers in similar fields will, no doubt, be interested in this discovery.

Department of Bio-Chemistry

$$
\text { M. Sreenivasaya. }
$$

Indian Institute of Science, Bangalore, Aug. 25.

\section{The Existence of the Cellobiose Residue in Cellulose.}

The chemical evidence for the view that cellobiose is preformed in cellulose is considerably strengthened by some observations we have made on the acetolysis of trimethyl cellulose. Under mild conditions of treatment at low temperatures fully methylated cellulose suffers cleavage to give a diacetyl-hexamethyl cellobiose, which is readily transformed into crystalline heptamethyl $\beta$-methylcellobioside. The experimental conditions under which this derivative of cellobiose is isolated preclude its occurrence as a reversion product of the reaction. Moreover, the yield of the crystalline $\beta$-cellobioside is equal to that of cellobiose octa-acetate obtained by the direct acetolysis of cellulose.

W. N. HaWORTH.

E. L. Hirst.

University, Edgbaston,

Birmingham. Aug. 18.

No. 3177 , VoL. 126] 